\title{
SOME APPLICATIONS OF THE POINCARÉ-BENDIXSON THEOREM
}

\author{
ROBERT ROUSSARIE
}

\begin{abstract}
We consider a $\mathcal{C}^{1}$ vector field $X$ defined on an open subset $U$ of the plane, with compact closure. If $X$ has no singular points and if $U$ is simply connected, a weak version of the Poincaré-Bendixson Theorem says that the limit sets of $X$ in $U$ are empty but that one can defined non empty extended limit sets contained into the boundary of $U$. We give an elementary proof of this result, independent of the classical Poincaré-Bendixson Theorem.

A trapping triangle $\mathcal{T}$ based at $p$, for a $\mathcal{C}^{1}$ vector field $X$ defined on an open subset $\mathcal{U}$ of the plane, is a topological triangle with a corner at a point $p$ located on the boundary $\partial \mathcal{U}$ and a good control of the tranversality of $X$ along the sides. The principal application of the weak Poincaré-Bendixson Theorem is that a trapping triangle at $p$ contains a separatrix converging toward the point $p$. This does not depend of the properties of $X$ along $\partial \mathcal{U}$. For instance, $X$ could be non differentiable at $p$, as in the example presented in the last section.
\end{abstract}

\section{INTRODUCTION}

We consider a $\mathcal{C}^{1}$ vector field $X$ on an open subset $U \subset \mathbb{R}^{2}$. This vector field is integrable with a $\mathcal{C}^{1}$ flow $\varphi(t, m)$ : for each $m \in U$, the map $t \mapsto \varphi(t, m)$ is the maximal trajectory with initial condition $\varphi(0, m)=m$ and is defined for $t \in\left(\tau_{-}(m), \tau_{+}(m)\right)$, interval whose end points verify $-\infty \leq \tau_{-}(m)<0<\tau_{+}(m) \leq+\infty$.

Of primordial importance is to know the future of the trajectory when $t \rightarrow \tau_{+}(m)$ and its past when $t \rightarrow \tau_{-}(m)$. To this end, one introduces the limit sets $\omega(m), \alpha(m)$ of $X$ defined by:

$$
\omega(m)=\left\{p \in U \mid \exists\left(t_{n}\right) \rightarrow \tau_{+}(m), \text { such that }\left(\varphi\left(t_{n}, m\right)\right) \rightarrow p\right\},
$$

and

$$
\alpha(m)=\left\{p \in U \mid \exists\left(t_{n}\right) \rightarrow \tau_{-}(m), \text { such that }\left(\varphi\left(t_{n}, m\right)\right) \rightarrow p\right\},
$$

The image of the trajetory: $\gamma=\gamma_{m}=\varphi\left(\left(\tau_{-}(m), \tau_{+}(m)\right), m\right)$ is the orbit of $m$. The limit sets depend just of $\gamma$ and we can them: $\omega(\gamma), \alpha(\gamma)$. More precisely, if $\gamma_{m}^{+}=\varphi\left(\left[0, \tau_{+}(m)\right), m\right)$ is the positive half-orbit and $\left.\gamma_{m}^{-}=\varphi\left(\left(\tau_{-}(m)\right), 0\right], m\right)$ the negative half-orbit, $\omega(m)$ depends just of $\gamma_{m}^{+}$ and $\alpha(m)$ just of $\gamma_{m}^{-}$. See [4], [6] for more information.

A description of the possible limit sets was given by Poincaré in [11] and this result was proved by Bendixson in [1]. More recent proofs of the so-called Poincaré-Bendixson Theorem may be found in [7], [9]. The following version appeared in [6]:

Theorem 1.1. (Poincaré-Bendixson Theorem) Let $X$ be a $\mathcal{C}^{1}$ vector field $X$ defined on an open set $U \subset \mathbb{R}^{2}$. One assumes that the singular points of $X$ are isolated. One considers a point $m \in U$ such that $\gamma_{m}^{+}$is contained into a compact subset of $U$. Then $\omega(m)$ is a singular point, a periodic orbit or a graphic: a topological immersion of the circle $S^{1}$, union of a finite number

2010 Mathematics Subject Classification. Primary: 34C05 ; Secondary: 34A26.

Key words and phrases. weak Poincaré-Bendixson Theorem, extended limit sets, trapping triangles, separatrix. 
of regular orbits connecting a finite number of singular points (see Figure 1). A similar result stands for the $\alpha$-limit sets.
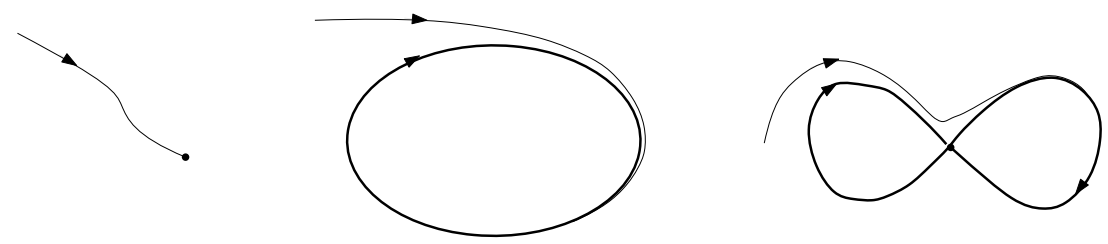

Figure 1. Possible $\omega$-limit sets

Remark 1.2. At least for real analytic vector fields, it is possible to extend Theorem 1.1 to vector fields with non-isolated singularities (see [10]).

If $\gamma_{m}^{+}$is not contained in a compact subset of $U$, it may happen that $\omega(m)$ be empty. But, if $\bar{U}$ is compact, it is always possible to extract from any sequence $\left(\varphi\left(t_{n}, m\right)\right)$ defined as above, a subsequence converging toward a point of $\bar{U}$. Then, in case that $\bar{U}$ is compact, it is natural to extend the definition of the limit sets by taking the limit of sequences $\left(\varphi\left(t_{n}, m\right)\right)$ in $\bar{U}$. In this way, one defined what one calls here : the extended limit sets $\bar{\omega}(m)$ and $\bar{\alpha}(m)$ (see Definition 2.1). These extended limit sets are always non empty.

Using this notion of extended limit sets, Theorem 1.3 below gives conditions on $U$ such that the limit sets in $\bar{U}$ are always contained in $\partial U$ (or equivalently, such that the usual limit sets in $U$ are empty). We call this result: weak Poincaré-Bendixson Theorem, because it can be seen in some cases as a rather trivial particular case of the Theorem 1.1. In fact we will give in Section 3 a simple proof of it, independent of the Theorem 1.1.

Theorem 1.3. (Weak Poincaré-Bendixson Theorem) One considers a $\mathcal{C}^{1}$ vector field $X$ defined on an open subset $U$ of $\mathbb{R}^{2}$, with a compact closure $\bar{U}$ We also assume that $X$ has no singular points and that $U$ is simply connected. Let $m$ be a point in $U$. Then, the limit sets $\omega(m)$ and $\alpha(m)$ are empty or equivalently the extended limit sets $\bar{\omega}(m)$ and $\bar{\alpha}(m)$ are contained into the boundary $\partial U=\bar{U} \backslash U$ (see Definition 2.1).

Remark 1.4. If one drops the condition that $X$ has no singular points or that $U$ is simply connected, it is very easy to find examples of extended limit sets not contained into $\partial U$.

Remark 1.5. In Theorem 1.3 we consider the closure $\bar{U}$ in $\mathbb{R}^{2}$. To say that $\bar{U}$ is compact is equivalent to say that $U$ is bounded. We could consider the closure in any compactification of $\mathbb{R}^{2}$ and prove the same result as Theorem 1.3, with exactly the same proof. Taking the closure in a compactification of $\mathbb{R}^{2}$ would allow for instance to study trajectories in $U$ with a limit set at infinity. It may be observed that there are different possible compactifications. The more usual compactifications in geometry are the Alexandroff compactification where $\mathbb{R}^{2}$ is identified to $\mathbb{C} \backslash\{\infty\}$ and the Lyapunov-Poincaré compactification where $\mathbb{R}^{2}$ is identified with the interior of the trigonometric disk (see [6]). In this paper, one is just interested in limit sets at finite distance. Then, Theorem 1.3 is just stated for bounded open sets.

The principal interest of Theorem 1.3 is that there is no assumption on the vector field $X$ along $\partial U$. For instance, $X$ could be non differentiable at some points of $\partial U$ and the classical 
Poincaré-Bendixson Theorem could not be applied in neighborhoods of such points. In Section 5 , we will present an example of vector field with a non-differentiable singular point, which stems from combustion theory (see [2]). Theorem 1.3 can be applied by putting the singular point at the boundary of the domain of study.

The extended limit sets are defined in Section 2, where some properties are given and where they are compared with the usual limit sets. In section 3, we give the proof of Theorem 1.3. This very simple proof is based on the non-recurrence property verified by a vector field without singular points, defined on a simply connected open set $U$. In Section 4 we present some applications of Theorem 1.3. The most important one is the notion of trapping triangle which gives conditions to have a trajectory tending toward a point of the boundary.

This is illustrated in some details in Section 5 where we recall how trapping triangles can be used to obtain interesting properties for a non-differentiable vector field introduced in [2]. The appendix is devoted to a sketch of proof of the Jordan-Schoenflies Theorem for $\mathcal{C}^{1}$ vector fields. This theorem enters as a key argument in the proof of Theorem 1.3.

\section{Extended Limit SETS}

In the whole section we assume that $X$ is a $\mathcal{C}^{1}$ vector field, defined on an open set $U$ of $\mathbb{R}^{2}$ with a compact closure $\bar{U}$.

Definition 2.1. (Extended limit sets) The $\bar{\omega}$-limit set of $m$ (in $\bar{U}$ ) is the compact subset of $\bar{U}$ defined as:

$$
\bar{\omega}(m)=\left\{p \in \bar{U} \mid \exists\left(t_{n}\right) \rightarrow \tau_{+}(m) \text { such that } \varphi\left(t_{n}, m\right) \rightarrow p\right\} .
$$

The $\bar{\alpha}$-limit set $\alpha(m)$ (in $\bar{U}$ ) is the $\bar{\omega}$-set of $m$, (in $\bar{U}$ ), for the field $-X$ (we have just to replace $\tau_{+}(m)$ by $\tau_{-}(m)$ in the above definition).

Remark 2.2. Extended limit sets limit sets $\bar{\omega}(m), \bar{\alpha}(m)$ are different from the usual ones, as we consider the limit values in $\bar{U}$ and not in $U$. It is the reason why we call them extended limit sets. We will write $\omega(m), \alpha(m)$ for the usual limit sets in $U$. Clearly, one has that $\omega(m)=\bar{\omega}(m) \cap U$ and $\alpha(m)=\bar{\alpha}(m) \cap U$. In fact, in Theorem 1.3 we are interested in a situation where the usual limit sets are empty, or equivalently where the extended limit sets are contained into $\partial U$.

It is easy to see that the extended limit sets are non-empty compact subsets of $\bar{U}$. The more important property is that they are limit of the trajectory of $m$ in the Haudorff sense, for positive or negative time. We recall that the Hausdorff distance between a point $p$ and a non-empty compact subset $A$ of $\mathbb{R}^{2}$ is given by:

$$
\operatorname{dist}_{H}(p, A)=\operatorname{Inf}\{\|m-p\||| m \in A\},
$$

where $\|\cdot\|$ is the Euclidean norm of $\mathbb{R}^{2}$. One has the following result:

Lemma 2.3. For $m \in U: \operatorname{dist}_{H}(\varphi(t, m), \bar{\omega}(m)) \rightarrow 0$ when $t \rightarrow \tau_{+}(m)$. There is a similar result for $\bar{\alpha}(m)$ when $t \rightarrow \tau_{-}(m)$.

Proof. We have just to consider the case of $\bar{\omega}(m)$. Assume that $\operatorname{dist}(\varphi(t, m), \bar{\omega}(m)) \nrightarrow \rightarrow 0$. This means that there exist $\varepsilon_{0}>0$ and a sequence $\left(t_{n}\right) \rightarrow \tau_{+}(m)$ such that $\forall n$ one has that $\varphi\left(t_{n}, m\right) \in$ $K\left(\varepsilon_{0}\right)=\left\{p \in \bar{U} \mid \operatorname{dist}(p, \bar{\omega}(m)) \geq \varepsilon_{0}\right\}$. As $K\left(\varepsilon_{0}\right)$ is compact, we can extract a subsequence $\left(t_{i}^{\prime}\right)=\left(t_{n_{i}}\right)$ such that the sequence $\left(\varphi\left(t_{i}^{\prime}, m\right)\right)$ converges toward some point $p_{0} \in K\left(\varepsilon_{0}\right)$, while $\left(t_{i}^{\prime}\right) \rightarrow \tau_{+}(m)$. This point $p_{0}$ belongs to $\bar{\omega}(m)$. We arrive to a contradiction, as $\operatorname{dist}_{H}\left(p_{0}, \bar{\omega}(m)\right) \geq$ $\varepsilon_{0}>0$ by passing to the limit. 
Remark 2.4. We will use the following particular case of Lemma 2.3: to say that $\bar{\omega}(m)$ is reduced to a single point $p$ is equivalent to say that $\varphi(t, m) \rightarrow p$ when $t \rightarrow \tau_{+}(m)$.

With a similar proof as in Lemma 2.3, one has the following:

Lemma 2.5. The extended limit sets are connected subsets of $\bar{U}$.

\section{Proof of the weak Poincaré-Bendixson Theorem}

We want to present a direct and simple proof, independent of the classical Poincaré-Bendixson Theorem. In fact this proof will just use an easy form of the non-existence of non-trivial recurrence property, adapted to the context. It is given in Lemma 3.1. Of course, the non-trivial recurrence property is also a key ingredient in the proof of the classical Poincaré-Bendixson Theorem (see [7] or [9] for instance). One has the following:

Lemma 3.1. Let $X$ be a $\mathcal{C}^{1}$ vector field defined on a simply connected open set $U$, without singular points. Then, an orbit of $X$ has at most one intersection point with an open transverse section to $X$, contained into $U$.

Proof. Let $\gamma$ be an orbit of $X$ and $\Sigma \subset U$ be an open transverse section to $X$. Assume that $\gamma \cap \Sigma$ contains at least two points. Let $p, q$ be two such points, consecutive on $\gamma$. We denote by $\gamma(p, q)$ the closed segment of orbit between $p, q$ and and by $\Sigma(p, q)$ the closed segment on $\Sigma$ between $p, q$. As the points $p, q$ are consecutive on $\gamma$, one has that $\gamma(p, q) \cap \Sigma(p, q)$ is the set with two points $\{p, q\}$. This means that $\Gamma=\gamma(p, q) \cup \Sigma(p, q)$ is a $\mathcal{C}^{1}$-piecewice simple curve in $U$. It is easy to smooth $\Gamma$ in order to obtain a $\mathcal{C}^{1}$ curve $\tilde{\Gamma}$, transverse to $X$. This curve can be chosen $\mathcal{C}^{0}$ arbitrarily near $\Gamma$, and then contained into $U$ (see Figure 2 : the segment of orbit $\gamma(p, q)$ is replaced by a transverse arc of curve $\tilde{\gamma}\left(p^{\prime}, q\right)$ inside a slim flow box $T$ along $\gamma(p, q)$; this flow box is a curved rectangle with corners the points $p, q, q^{\prime}, p^{\prime}$; a detailled proof can be found in [12]). It follows from the $\mathcal{C}^{1}$ Jordan-Schoenflies Theorem that this curve bounds a topological disk $\tilde{D} \subset U$. As $X$ is transverse to $\partial \tilde{D}=\tilde{\Gamma}$, the disk $\tilde{D}$ must contain a singular point of $X$, as it follows for instance from the Brouwer Theorem. We are arrived to a contradiction.

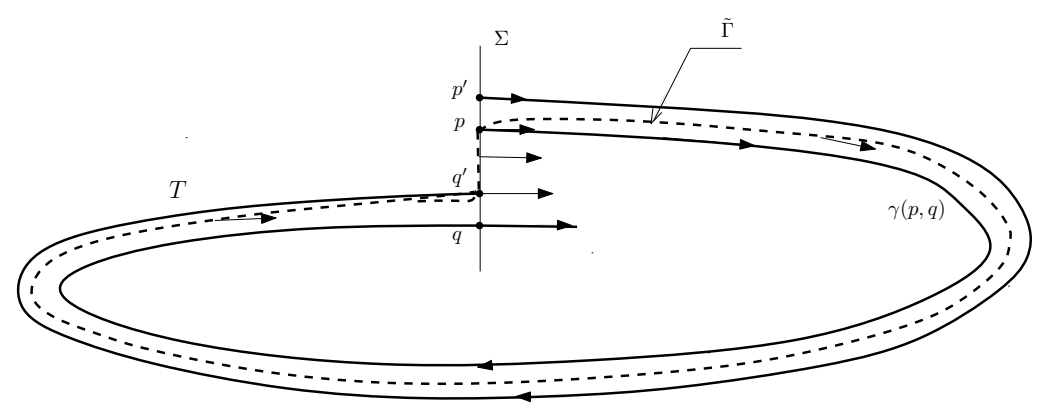

Figure 2. Construction of $\tilde{\Gamma}$

Remark 3.2. Jordan-Schoenfies Theorem is true for any topological closed curve embedded into $\mathbb{R}^{2}$, also called Jordan curve. This theorem says that there is an homeomorphism of $\mathbb{R}^{2}$ to itself sending the trigonometric circle $\Gamma_{0}$ onto $\Gamma$. As a consequence, $\Gamma$ bounds a topological disk 
contained in any neighborhood $U$ of $\Gamma$. The proof in this general $\mathcal{C}^{0}$ context is rather delicate (nevertheless, one can see [3] for an elementary proof). The proof is much easier for a $\mathcal{C}^{1}$ closed curve $\Gamma$. As it is the only case used in this paper, a sketch of proof is given in appendix.

Remark 3.3. One can find in [8] an easy topological proof of the Poincaré-Hopf formula, whose Brouwer Theorem is a particular case.

Lemma 3.1 easily implies that:

Proof of Theorem 1.3: Consider a point $m \in U$. We have just to prove that $\bar{\omega}(m) \subset \partial U$ : the proof for $\bar{\alpha}(m)$ is the same, changing $X$ by $-X$.

Let us assume on contrary, that a point $p \in \bar{\omega}(m)$ belongs to $U$. There exists a sequence $\left(t_{n}\right) \rightarrow \tau_{+}$such that $\varphi\left(t_{n}, m\right) \rightarrow p$. By hypothesis this point is regular: $(X(p) \neq 0)$. Take a flow-box $W$ in $U$, diffeomorphic to a closed rectangle $\Sigma \times I$, centered at $(0,0) \in \mathbb{R}^{2}$, where $p=(0,0)$ and $\Sigma=\Sigma \times\{0\}$ is a transverse section to $X$. For $n$ large enough, it is easy to change sligthly $t_{n}$ in order that $\varphi\left(t_{n}, m\right) \in \Sigma$. As a consequence the half-orbit $\gamma_{+}(m)$ cuts $\Sigma$ in infinitely many points. This contredicts Lemma 3.1. Then, one has that $\omega(m)=\bar{\omega}(m) \cap U=\emptyset$, i.e. that $\bar{\omega}(m) \subset \partial U$.

\section{Applications of the weak Poincaré-Bendixson Theorem}

Applications of the weak Poincaré-Bendixson Theorem depend on the properties we will assume for the vector field $X$ on the boundary of $U$. It follows from Lemma 2.5 that an extended limit set is a compact connected subset of $\partial U$. Then, if $\partial U$ is a topological curve, an extended limit set is an isolated point or is homeomorphic to a closed interval. This last possibility may occur when the properties of $X$ are rather wild near the boundary. For instance, consider the Hamiltonian vector field $X_{H}$ of Hamiltonian function $H(x, y)=y-x \sin x$. This Hamiltonian vector field has no singular points in the whole plane. Each trajectory oscillates indefinitely between a pair of lines $\{y \pm x=$ Const. $\}$ (see Figure 3). If we take the direct image of $X_{H}$ by a smooth diffeomorphism of $\mathbb{R}^{2}$ onto the open disk $U$ of radius 1 , preserving the radial directions, we obtain a smooth vector field on $U$, whose each limit set is one of the intervals $\left\{-\frac{\pi}{2} \leq \theta \leq+\frac{\pi}{2}\right\}$ or $\left\{\frac{3 \pi}{2} \leq \theta \leq+\frac{5 \pi}{2}\right\}$ on the trigonometric circle.

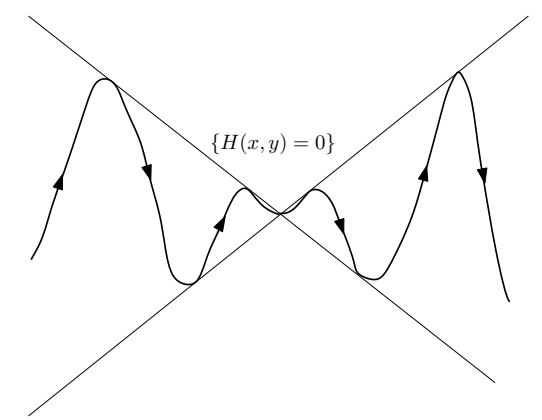

Figure 3. The orbit $\{H(x, y)=0\}$

The above example is of course rather pathological. We are more interested in finding conditions such that a trajectory in $U$ tends toward a point of the boundary $\partial U$. There is a circonstance when this occurs rather trivially: 
Lemma 4.1. Let $X$ be a $\mathcal{C}^{1}$ vector field defined on an open subset $U$ of $\mathbb{R}^{2}$ with a compact closure. Let $p$ be a point in $\bar{\omega}(m) \cap \partial U$, for some $m \in U$. One assumes that $X$ can be extended in a neighbohood $W$ of $p$ in $\mathbb{R}^{2}$ as a $\mathcal{C}^{1}$ vector field, still be called $X$. One also assumes that $X(p) \neq 0$ is transverse to $\partial U$, supposed to be a regular $\mathcal{C}^{1}$ curve in a neighborhood of $p$. Then $\bar{\omega}(m)=\{p\}$ and the trajectory of the extended vector field $X$ arrives to $p$ at the time $\tau_{+}(m)$, the finite positive limit time of the trajectory in $U$.

Proof. We choose $W$ to be a flow box of the extended vector field $X$, diffeomorphic to $\left[-\varepsilon_{0}, \varepsilon_{0}\right] \times$ $\left[-\delta_{0}, \delta_{0}\right]$, where $p=(0,0)$. The intervals $\left[-\varepsilon_{0}, \varepsilon_{0}\right] \times\{\delta\}$ are segments of orbits and intervals $\{\varepsilon\} \times\left[-\delta_{0}, \delta_{0}\right]$ are transverse sections, for all $(\varepsilon, \delta) \in\left[-\varepsilon_{0}, \varepsilon_{0}\right] \times\left[-\delta_{0}, \delta_{0}\right]$. As $p \in \bar{\omega}(m)$ there is a time $t_{0}$ such that $\varphi\left(t_{0}, m\right) \in W$, and more precisely $\varphi\left(t_{0}, m\right)=\left(\varepsilon_{t_{0}}, 0\right)$ for some $\varepsilon_{t_{0}} \in\left[\varepsilon_{0}, 0\right)$. Then, for all $t \geq t_{0}, \varphi(t, m)$ must be also a point of the same type $\left(\varepsilon_{t}, 0\right)$ for some $\varepsilon_{t} \in\left[\varepsilon_{t_{0}}, 0\right)$. The conclusions of the lemma clearly follow.

4.1. Large flow boxes. Let $X$ be a $\mathcal{C}^{k}$ vector field, with $k \geq 1$. The usual Flow-Box Theorem gives a normal form for $X$ in a neighborhood of any regular point: if $m$ is such that $X(m) \neq 0$, there exists a neighborhood $W$ of $(0,0) \in \mathbb{R}^{2}$ (with coordinates $(x, y)$ ) and a $\mathcal{C}^{k}$ diffeomorphism $\Phi$ sending the vector field $\frac{\partial}{\partial x}$ onto the vector field $X$. $\mathcal{T}=\Phi(W)$ is called a flow box of $X$. Using Theorem 1.3, we can prove the following:

Proposition 4.2. Let $X$ be a $\mathcal{C}^{k}$ vector field, with $k \geq 1$, defined on an open set $\mathcal{U}$, without singular points. Let $\mathcal{T} \subset \mathcal{U}$ be a $\mathcal{C}^{k}$-piecewise rectangle. One assumes that $\mathcal{T}$ has two sides $[A, B],[D C]$ which are segments of orbits and the two sides $[A D],[B C]$ which are transverse sections such that $X$ is pointing inward $\mathcal{T}$ along $[A D]$ and outward $\mathcal{T}$ along $[B C]$ (see Figure 4). Then the trajectory starting at a point $m \in[A D]$ arrives at a point of $[B C]$ in a finite time $t(m)$. The function $t(m)$ is $\mathcal{C}^{k}$.

Proof. We can apply Theorem 1.3 by taking $U$ to be the interior of $\mathcal{T}$. If $m \in[A D]$, its trajectory passes through a nearby point $m^{\prime}$ located in $U$. It follows from Theorem 1.3 that $\bar{\omega}\left(m^{\prime}\right) \in \partial \mathcal{T}$. As $\bar{\omega}\left(m^{\prime}\right)$ cannot contain points of the open arcs of trajectory $] A B[$ and $] D C[$, nor points of $[A, B]$ because $X$ is entering along this side, we have that $\bar{\omega}\left(m^{\prime}\right) \subset[B C]$. It follows from Lemma 4.1 that $\bar{\omega}\left(m^{\prime}\right)$ is just a point of $[B C]$ and as a consequence, there is a finite time $t(m)$ such that $\varphi(t(m), m) \in[B C]$. As the trajectory of $m$ arrives transversally on $[B C]$, we can use the Cauchy Theorem in class $\mathcal{C}^{k}$ (saying that the flow is a $\mathcal{C}^{k}$ map) and the Inverse Function Theorem to show that $t(m)$ is a function of class $\mathcal{C}^{k}$.

In fact a rectangle as in Proposition 4.2 is a flow box. As its size is arbitrary, we can say that it is a large flow box. More precisely one has the following:

Corollary 4.3. Let $X, U$ and $\mathcal{T}$ and $t(m)$ as in Proposition 4.2. One parametrizes $[A D]$ by $y \in$ $[0,1]$. Let $W$ be the curved rectangle of $\mathbb{R}^{2}$ defined by $W=\left\{(x, y) \in \mathbb{R}^{2} \mid y \in[0,1], x \in[0, t(y)]\right\}$. Then, the map $(x, y) \mapsto \varphi(x, y)$ is a $\mathcal{C}^{k}$ diffeomorphism from $W$ onto $\mathcal{T}$, sending $\frac{\partial}{\partial x}$ on $X$.

Proof. We identify $[A D]$ with the interval $[0,1]$ parametrized by $y$. For any $y \in(01)$ the arc of orbit starting at $y$ is disjoint from the sides $[A B]$ and $[D C]$. The same argument than the one used in Proposition 4.2 shows that if $y \neq y^{\prime}$ are two points on $[A D]$, then the arcs of trajectories in $\mathcal{T}$, starting at $y$ and $y^{\prime}$ are disjoint. As the flow is injective on each arc, one has that the map $(x, y) \mapsto \varphi(x, y)$ is one-to-one from $W$ onto $\mathcal{T}$. As a consequence of the Cauchy 


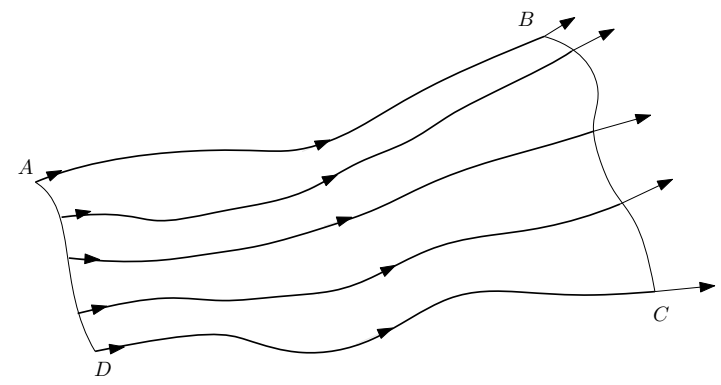

Figure 4. A large flow box

Theorem, this map is $\mathcal{C}^{k}$. As $D \varphi(x, y)\left[\frac{\partial}{\partial x}\right]=X(\varphi(x, y))$ and as $D \varphi(x, y)\left[\frac{\partial}{\partial y}\right]$ is a vector tranverse to $X(\varphi(x, y))$, the map $(x, y) \rightarrow \varphi(x, y)$ has a maximal rank at each $(x, y) \in W$. Then, this map is a $\mathcal{C}^{k}$ diffeomorphism from $W$ onto $\mathcal{T}$. Finally, as $D \varphi(x, y)\left[\frac{\partial}{\partial x}\right]=X(\varphi(x, y))$, this map sends the vector field $\frac{\partial}{\partial x}$ on the vector field $X$.

4.2. Closed nodal region. We consider a vector field $X$ on an open set $\mathcal{U}$. We assume that $X$ has a singular point $O$ in $\mathcal{U}$ and that $X$ is $\mathcal{C}^{1}$ in $\mathcal{U} \backslash\{O\}$. The following notion was introduced by Bendixson in [1]:

Definition 4.4 (Bendixson). A closed nodal region at $O$ for $X$ is a topological disk $D(\Gamma)$ pinched at a singular point $O$, bounded by an orbit $\Gamma$ such that $\omega(\Gamma)=\alpha(\Gamma)=\{O\}$ and filled by orbits with the same limit property (see Figure 5).

The following result was proved by Bendixson in [1]:

Lemma 4.5. One considers a vector field $X$ on a simply connected open set $\mathcal{U}$. One assumes that $X$ has a unique singular point $O$ in $\mathcal{U}$ and that $X$ is $\mathcal{C}^{1}$ in $\mathcal{U} \backslash\{O\}$. One also assumes that $X$ is a has an orbit $\Gamma$ such that $\omega(\Gamma)=\alpha(\Gamma)=\{O\}$. Then $\Gamma \cup\{O\}$ is the boundary in $\mathcal{U}$ of a topological disk which is closed nodal region at $O$ for $X$.

Proof. It follows from the Jordan-Schoenflies Theorem that $\Gamma \cup\{O\}$ is the boundary of a topological disk $D$ in $\mathcal{U}$. As the interior $U$ of this disk $D$ contains no singular point, we can apply Theorem 1.3 to it: the extended limit sets of any point of $U$ are contained into $\partial D=\Gamma \cup\{O\}$. As these limit sets cannot contain any point of $\Gamma$, they are reduced to $\{O\}$. 


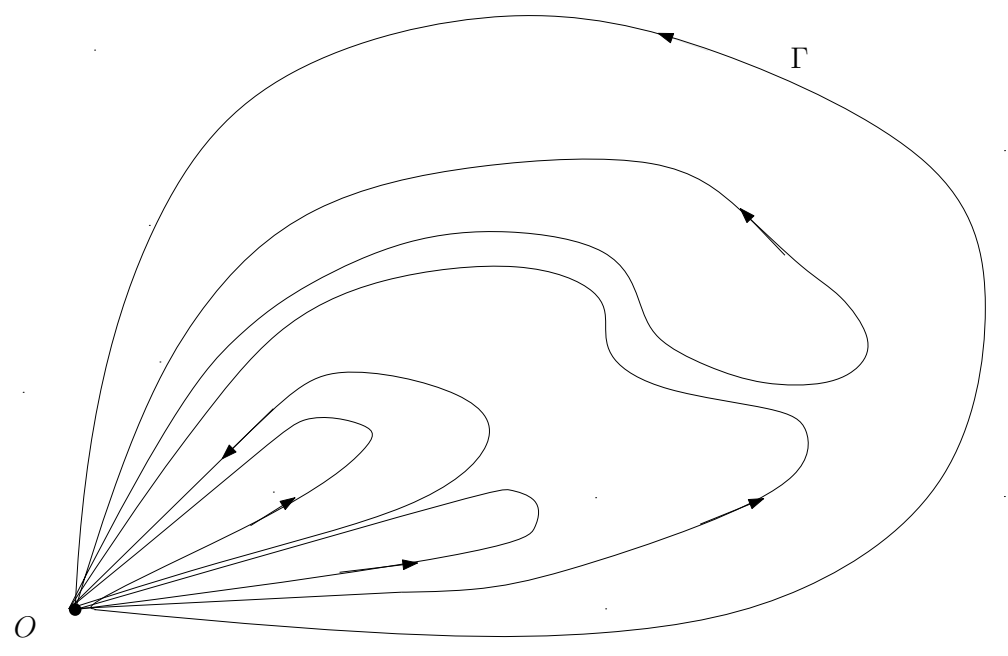

Figure 5. Closed nodal region

Remark 4.6. Elliptic sectors, used in the classification of the phase portrait of vector field near an isolated singular point (see [5], [12]), are simple examples of closed nodal regions. We can introduce an order in the set of orbits contained into $D(\Gamma)$, putting that $\tilde{\Gamma}_{1}$ is less than $\tilde{\Gamma}_{2}$ if and only if $\tilde{\Gamma}_{1} \subset D\left(\tilde{\Gamma}_{2}\right)$. This order is total for an elliptic sector but is just partial in general. As a consequence, the phase portrait inside a general closed nodal region may be much more complicated than the simple 1-parameter family of orbits that one finds inside an elliptic sector. See Figure 5 for an example of a closed nodal region which is not an elliptic sector.

4.3. Trapping triangles. We consider a vector field $X$ defined on an open subset $\mathcal{U}$ of $\mathbb{R}^{2}$, with closure $\overline{\mathcal{U}}$ not necessarily compact. We assume that $X$ is $\mathcal{C}^{1}$ on $\mathcal{U}$, but nothing is said about a possible extention of $X$ along the boundary $\partial \mathcal{U}=\overline{\mathcal{U}} \backslash \mathcal{U}$. We look for conditions which could ensure that a trajectory in $\mathcal{U}$ converges toward some point $p \in \partial \mathcal{U}$. One will use the following:

Definition 4.7. A trapping triangle $\mathcal{T}=[p q r]$ at $p \in \partial \mathcal{U}$, for the vector field $X$, is a topological triangle contained into $\overline{\mathcal{U}}$, with corners $p, q, r$ such that $\mathcal{T} \cap \partial \mathcal{U}=\{p\}$ (or equivalently $\mathcal{T} \backslash\{p\} \subset$ $\mathcal{U})$. This triangle has three sides $[p q],[p, r]$ and $[q r]$. The arcs $(p q],(p, r]$ and $[q r]$ are $\mathcal{C}^{1}$ regular arcs (i.e. contained into regular open curves of class $\mathcal{C}^{1}$ ). One assumes that $X$ is tranverse to $(p, q],[r, p)$ and $[q r]$. Moreover, one assumes that $X$ points outside $\mathcal{T}$ along $(p, q),(p, r)$ and inside $\mathcal{T}$ along $(q, r)$ (see Figure 6$)$. 


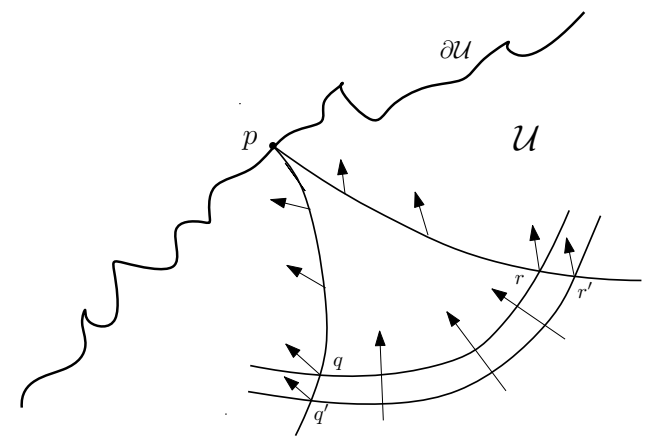

FiguRE 6. Trapping triangle

As a consequence of Theorem 1.3, one has the following result:

Lemma 4.8. Let $\mathcal{T}=[p q r]$ be a trapping triangle as in Definition 4.7. There exist points $m \in$ $(q, r)$ whose trajectory $\varphi(t, m)$ remains into $\mathcal{T}$ for all times and tends toward $p$ for $t \rightarrow \tau^{+}(m)$. Trajectories starting at other points of $(q, r)$ cut the side $(p, q]$ or the side $(p, r]$ after a finite time.

Proof. We begin by extending slightly the triangle $\mathcal{T}$ into a new triangle $\mathcal{T}^{\prime}=\left[p q^{\prime} r^{\prime}\right]$, with the same properties than $\mathcal{T}$, by taking a $\mathcal{C}^{1}$ regular arc $\left[q^{\prime}, r^{\prime}\right]$ near $[q, r]$ (see Figure 6 ). We call $U$ the interior of $\mathcal{T}^{\prime}$. It is clear that the pair $(U, X)$ verifies the statements of Theorem 1.3 and one considers $X$ restricted to $U$ in the rest of the proof. Let $m$ be a point of $(q, r)$. As $(q, r) \subset U$, by Theorem 1.3 one has that $\bar{\omega}(m) \subset\left[p, q^{\prime}\right] \cup\left[p, r^{\prime}\right] \cup\left[q^{\prime}, r^{\prime}\right]$. As a consequence of the direction of $X$ along $\left(q^{\prime}, r^{\prime}\right)$, no point of $\bar{\omega}(m)$ can belong to $\left(q^{\prime}, r^{\prime}\right)$. Then, $\bar{\omega}(m) \subset\left[q^{\prime}, p\right] \cup\left[p, r^{\prime}\right]$.

By Lemma 4.1, we know that, if $\bar{\omega}(m)$ contains a point $a$ of $\left[q^{\prime}, p\right] \cup\left[p, r^{\prime}\right] \backslash\{p\}$ then, $\bar{\omega}(m)=$ $\{a\}$. For the same reason, if $\bar{\omega}(m)$ contains the point $p$, it cannot contain any other point in $\left[q^{\prime}, p\right] \cup\left[p, r^{\prime}\right]$ and then its is reduced to $\{p\}$. It follows that one has just three possibilities: $\bar{\omega}(m)$ is a point in $\left(p, q^{\prime}\right]$, is a point of $\left(p, r^{\prime}\right]$ or is the point $p$. The set $\mathcal{O}_{q}$ of the points of $(q, r)$ whose $\bar{\omega}$-set belongs to $\left(p, q^{\prime}\right]$ is a non empty open set of $(q, r)$. The reason is that, if $a$ is such a point, one has that $X(a) \neq 0$. Then $\tau_{+}(m)$ is finite and is a value attained by the flow of $X$ on $\mathcal{U}$. Moreover, as the trajectory is transverse at $a$ to regular curve $\left(p, q^{\prime}\right]$, these properties remain true for the points $m^{\prime}$ near $m$ on $(q, r)$ : the trajectory through $m^{\prime}$ attains also $\left(p, q^{\prime}\right]$ after a finite time. For the same reason, the set $\mathcal{O}_{r}$ of the points of $(q, r)$ whose $\bar{\omega}$-set belongs to $\left(p, r^{\prime}\right]$ is a non empty open set of $(q, r)$. As $(q, r)$ is connected $F_{p}=(q, r) \backslash \mathcal{O}_{q} \cup \mathcal{O}_{r}$ is non-empty. A point $m \in F_{p}$ is such that $\bar{\omega}(m)=\{p\}$. By Lemma 2.3 (see also Remark 2.4) this means that the trajectory of $m$ tends toward $p$ when $t \rightarrow \tau_{+}(m)$. It is clear that this trajectory remains into $U$.

It is possible to give more information about the phase portrait of $X$ in $\mathcal{T}$ :

Lemma 4.9. One assumes that $X$ is $\mathcal{C}^{k}$ for $1 \leq k \leq+\infty$. Let $\mathcal{T}=[p q r]$ be a trapping triangle as in Definition 4.7. The set of points of $(q, r)$ whose trajectory tends toward $p$ is a closed interval $\left[m_{0}, m_{1}\right] \subset(q, r)$, maybe reduced to a single point. The trajectory from $m \in\left(q, m_{0}\right)$ reaches $(q, p)$ after a finite time $t(m)$. Similarly, the trajectory from $m \in\left(m_{1}, r\right)$ reaches $(r, p)$ after a finite time $t(m)$ These functions $t(m)$ are $\mathcal{C}^{k}$ (see Figure 7). 


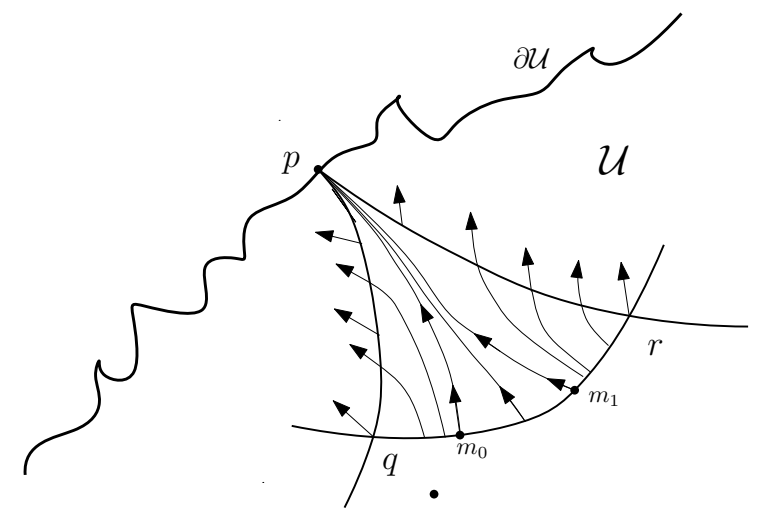

FiguRE 7. Dynamics inside the trapping triangle

Proof. We use the notations introduced in the proof of Lemma 4.8: $U$ is the interior of a larger triangle $\left[p q^{\prime} r^{\prime}\right]$ and $\mathcal{O}_{q}, \mathcal{O}_{r}, F_{p}$ the subsets of $(q, r)$ defined as above in the proof of Lemma 4.8. Let $m_{0}$ be the upper bound of points $m \in(q, r)$ such that $(q, m) \subset \mathcal{O}_{q}$. As $\mathcal{O}_{q}$ and $\mathcal{O}_{r}$ are open subsets of $(q, r)$ the point $m_{0}$ cannot belong to none of them. Then $m_{0} \in F_{p}$. In a similar way, we can find a point $m_{1} \in F_{p}$ associated to $\mathcal{O}_{r}$. Clearly we have that $m_{0} \leq m_{1}$ for the orientation going from $q$ to $r$. If $m_{0} \neq m_{1}$, we can apply Theorem 1.3 to the triangle $\left[p m_{0} m_{1}\right]$ with sides the positive half orbits $\gamma^{+}\left(m_{0}\right), \gamma^{+}\left(m_{1}\right)$ union their $\bar{\omega}$-limit $p$ and the subarc $\left[m_{0}, m_{1}\right]$ on $(q, r)$ : for any point $m \in\left(m_{0}, m_{1}\right)$ the limit set $\bar{\omega}(m)=\{p\}$, as this limit set cannot contains points of the regular orbits $\gamma^{+}\left(m_{0}\right), \gamma^{+}\left(m_{1}\right)$. Then, we have that $\left[m_{0}, m_{1}\right]$ is the set $F_{p}$ of points on $(q, r)$ whose trajectory tends toward $p$ and that $\mathcal{O}_{q}=\left(q, m_{0}\right), \mathcal{O}_{r}=\left(m_{1}, r\right)$. The fact that the function $t(m)$ is $\mathcal{C}^{k}$ follows from the Cauchy Theorem in class $\mathcal{C}^{k}$, saying that the flow map $(t, m) \mapsto \varphi(t, m)$ is $\mathcal{C}^{k}$, and the Inverse Function Theorem used to define implicitly the functions $t(m)$.

\section{How to USE TRAPPING TRIANGLES?}

Trapping triangles can be used in order to obtain the existence of a separatrix tending toward a singular point $p$. To this end one places this point at the boundary of an open set $\mathcal{U}$, shows that there exists a trapping triangle at $p$ and applies Lemma 4.8. Next, by chosing convenient other trapping triangles, it may be possible to obtain more precisions about the detected separatrix. This method uses principally qualitative arguments with a minimum of computations which are in general rather direct. Moreover, one can apply the method to a vector field not differentiable for instance at the point $p$. In such a case, it is not possible to apply the classical PoincaréBendixson Theorem in a neighborhood of $p$.

We would like to illustrate this method with an example presented in a recent paper (see [2]), which deals with a free interface problem in combustion theory. More specifically, one considers a system of two reaction-diffusion equations that models diffusional-thermal combustion with ignition-temperature kinetics and fractional order $\alpha$. Looking for special solutions, namely onedimensional traveling waves, turns out to be equivalent to finding a trajectory tending towards 
the origin for the vector field $X_{c}$ with differential equation :

$$
\left\{\begin{array}{l}
x^{\prime}=y, \\
y^{\prime}=\frac{1}{\Lambda}\left(c y+x^{\alpha}\right) .
\end{array}\right.
$$

Moreover, this trajectory must verify the initial conditions: $x(0)=v_{0}$ and $y(0)=-\frac{c}{\Lambda}\left(1-v_{0}\right)$. Here, on the one hand, $c>0$ is the speed of the traveling wave (to be determined), $v_{0} \in[0,1]$ is fixed at this phase of the study; on the other hand, $\Lambda>0$ (the inverse of the Lewis number) and $\alpha \in[0,1]$ are physical parameters. For instance, the parameter $\alpha$ can change with the ratio of the two reactants and may take non integer values. For such values of $\alpha$ the vector field $X_{c}$ is just defined for $x \geq 0$ and is not differentiable along the axis $\{x=0\}$.

For physical reasons, the vector field $X_{c}$ is considered in the quadrant $Q=\{x \geqslant 0, y \leqslant 0\}$. For $c=0$, the vector field $X_{0}$ is Hamiltonian with Hamiltonian function

$$
H(x, y)=\frac{1}{2} y^{2}-\frac{1}{\Lambda(1+\alpha)} x^{1+\alpha} .
$$

This vector field has in $Q$, a stable separatrix $L_{0}$ at the origin $O$ :

$$
L_{0}:=\left\{y=y_{0}(x)=-\left(\frac{2}{\Lambda(1+\alpha)}\right)^{1 / 2} x^{\frac{1+\alpha}{2}}\right\} .
$$

For any $c \geqslant 0$, we have that:

$$
X_{c} \cdot H(x, y)=-\frac{1}{\Lambda} y x^{\alpha}+\frac{1}{\Lambda}\left(c y+x^{\alpha}\right) y=\frac{c}{\Lambda} y^{2} .
$$

This implies that, for $c>0$, the vector field $X_{c}$ is transverse to $L_{0}$ and directed downwards all along $L_{0}$, outside $O$.

Now, for any $v_{0}>0$, we consider in $Q$ a trapping triangle $\mathcal{T}_{v_{0}}$. This triangle has three corners: $O, A_{v_{0}}=\left(v_{0}, 0\right)$ and $B_{v_{0}}=\left(v_{0}, y_{0}\left(v_{0}\right)\right)$; and three sides denoted as follows: $\left[O A_{v_{0}}\right]$ on the $0 x$ axis, $\left[O B_{v_{0}}\right]$ on the curve $L_{0}$ and $\left[A_{v_{0}} B_{v_{0}}\right]$. As the vector field $X_{c}$ is vertical at the point $A_{v_{0}}$, one chooses for side $\left[A_{v_{0}} B_{v_{0}}\right]$ a vertical segment in $\left\{x=v_{0}\right\}$ modified by a small bump near $A_{v_{0}}$, in order that $X_{c}$ was transverse along this side with a left direction. The vector field $X_{c}$ is transverse and has an upward direction along $\left(O A_{v_{0}}\right]=\left[O A_{v_{0}}\right] \backslash\{O\}$. As already mentioned, $X_{c}$ is transverse and has a downward direction along $\left(O B_{v_{0}}\right]=\left[O B_{v_{0}}\right] \backslash\{O\}$. (see Figure 8).

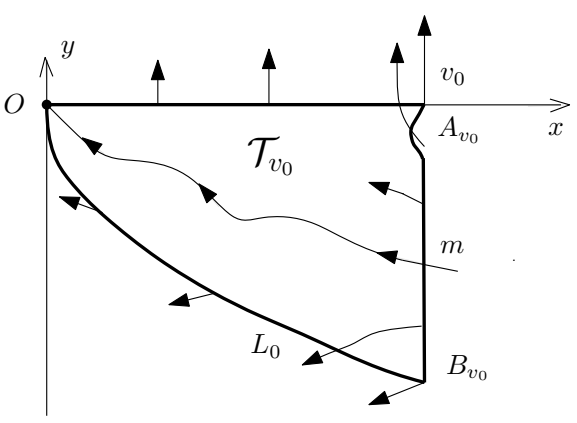

Figure 8. Trapping triangle $\mathcal{T}_{v_{0}}$ 
As consequence of Lemma 4.8, there exists an orbit $L_{c}$ of $X_{c}$ in $Q$ which tends toward $O$ and then is a stable separatrix at this singular point. Much more is obtained in [2] about $L_{c}$. This orbit is the unique one tending toward $O$. In the large, it is graph of a smooth function $y_{c}(x)$ defined for $x \in(0,+\infty)$ and which extends continuously by $y_{c}(0)=0$.

It is possible to use a finer trapping triangle $\mathcal{T}_{v_{0}}^{\prime}$ in order to obtain the following expression:

$$
y_{c}(x)=-\left(\frac{2}{\Lambda(1+\alpha)}\right)^{1 / 2} x^{\frac{1+\alpha}{2}}+o\left(x^{\frac{1+\alpha}{2}}\right) .
$$

To this end, we consider the curve:

$$
l_{c}:=\left\{y=\tilde{y}_{c}(x)=-\left(\frac{2}{(1+\alpha) \Lambda}\right)^{1 / 2} x^{\frac{1+\alpha}{2}}+\frac{c}{\Lambda} x\right\} .
$$

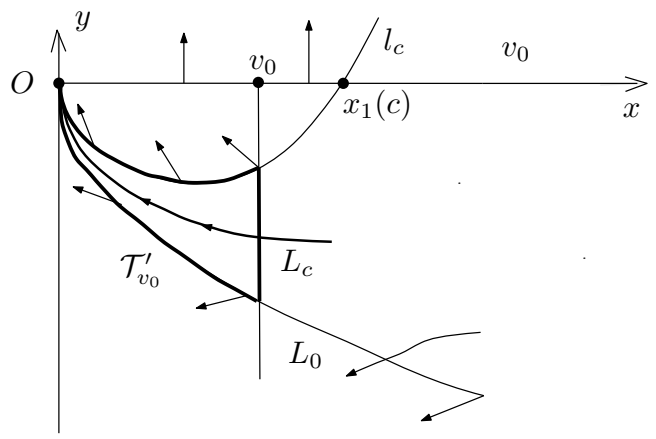

FIGURE 9. Trapping triangle $\mathcal{T}_{v_{0}}^{\prime}$

It is easy to see that, the vector field $X_{c}$ is transverse along $l_{c}$, with an upward direction (see [2] for the computation). One can observe that the curve $l_{c}$ cuts the $O x$-axis at the value $x_{1}(c)=\left(\frac{2 \Lambda}{(1+\alpha) c^{2}}\right)^{\frac{1}{1-\alpha}}>0$ and remains in the quadrant $Q$ only for $x \in\left[0, x_{1}(c)\right]$. Nevertheless, we can construct a new trapping triangle $\mathcal{T}_{v_{0}}^{\prime}$, using the curves $L_{0}$ and $l_{c}$, with a vertical side in $\left\{x=v_{0}\right\}$ when $0<v_{0}<x_{1}(c)$ (see Figure 9; as $v_{0}<x_{1}(c)$, one does not need now to modify the vertical side by a bump). The graph of $L_{c}$ is trapped inside $\mathcal{T}_{v_{0}}^{\prime}$. It follows that $y_{0}(x)<y_{c}(x)<\tilde{y}_{c}(x)$ for $0<x<v_{0}$ and these inequalities imply the asymptotic 5.3. In [2], it is also shown that for any $x>0$, the function $c \rightarrow y_{c}(x)$ is continuous and increasing. This allows to find a value $c\left(v_{0}\right)$ in order to fulfill the above initial conditions: $c\left(v_{0}\right)$ is the unique solution to the equation $y_{c}\left(v_{0}\right)=-\frac{c}{\Lambda}\left(1-v_{0}\right)$. These results are obtained using a new well-chosen trapping triangle.

It follows from 5.3 that the time $R\left(v_{0}\right)$ "to arrive at $O$ from the initial condition $\left(v_{0},-\frac{c}{\Lambda}(1-\right.$ $\left.v_{0}\right)$ )" is finite if $\alpha<1$ (said more correctly, $R\left(v_{0}\right)$ is the finite limit time $\tau_{+}\left(v_{0},-\frac{c}{\Lambda}\left(1-v_{0}\right)\right.$ ) for the vector field $\left.X_{c\left(v_{0}\right)}\right)$. Moreover, using estimations on $c\left(v_{0}\right)$ given in [2] (which may be also obtained by a qualitative arguement) and a new trapping triangle where the curve $l_{c}$ is replaced by a curve $d_{k}:=\left\{y=-k x^{\frac{1+\alpha}{2}}\right\}$ for a well-chosen $k<0$, one obtains the following inequalities:

$$
\frac{(2(1+\alpha) \Lambda)^{1 / 2}}{1-\alpha} v_{0}^{\frac{1-\alpha}{2}}<R\left(v_{0}\right)<\frac{4 \Lambda^{1 / 2}}{(1-\alpha)(1+\alpha)^{1 / 2}} \frac{v_{0}^{\frac{1-\alpha}{2}}}{1-v_{0}},
$$


for $0<v_{0}<1$ and $0 \leqslant \alpha<1$.

The value of $R\left(v_{0}\right)$ is directly related to the trailing interface at which the deficient reactant is completely comsumed in the combustion model. As explained in [2], the result that $R\left(v_{0}\right)$ is finite for $0 \leq \alpha<1$ and the inequalities (5.5) are highly significant for this problem.

Acknowledgment The author wants to thank Claude-Michel Brauner for the suggestion to write this article and the encouragements provided during this task.

\section{Appendix: The Jordan-Schoenflies Theorem in class $\mathcal{C}^{1}$}

We want to give a sketch of proof for the following restricted version of the Jordan-Schoenflies Theorem, the only version used in the present article. The support of a diffeomorphism $H$ of $\mathbb{R}^{2}$ is the support $H-I d$.

Theorem 5.1. (Jordan-Schoenflies Theorem in class $\mathcal{C}^{1}$ ) Let $\Gamma$ be a $\mathcal{C}^{1}$ closed regular curve in $\mathbb{R}^{2}$. There exists a $\mathcal{C}^{1}$ diffeomorphism $H$ of $\mathbb{R}^{2}$, with compact support, sending the trigonometric circle onto $\Gamma$.

Proof. We choose a tubular neighborhood $T$ of $\Gamma$ with a $\mathcal{C}^{1}$ trivialization $T \cong S^{1} \times[-1,+1]$ such that $S^{1} \times\{0\}$ corresponds to $\Gamma$. The segments $\{\theta\} \times[-1,+1]$ give a $\mathcal{C}^{1}$ normal fibration $\mathcal{N}$ along $\Gamma$. A first step is to approach $\Gamma$ by a $\mathcal{C}^{\infty}$ closed curve $\Gamma_{1}$ in the $\mathcal{C}^{1}$ topology. In a second step $\Gamma_{1}$ can be approached in the $\mathcal{C}^{\infty}$ topology by a smooth closed $\Gamma_{2}$ in generic position in relation with the foliation $\mathcal{F}$ by the horizontal lines $\{y=$ Const. $\}$, meaning that all contact points of $\Gamma_{2}$ with $\mathcal{F}$ are quadratic and located on different leaves (see Figure 10). We can choose $\Gamma_{2}$ sufficiently near $\Gamma$ in order that $\Gamma_{2}$ is inside the interior of $T$ and transverse to $\mathcal{N}$. Then, $\Gamma_{2}$ is given in the trivialization, by the graph of a map from $S^{1}$ to ] $-1,1\left[\right.$ and it is easy to construct a $\mathcal{C}^{1}$ diffeomorphism of $\mathbb{R}^{2}$, with support in $T$, sending each fiber of $\mathcal{N}$ onto itself and sending $\Gamma$ onto $\Gamma_{2}$ (see Figure 11).

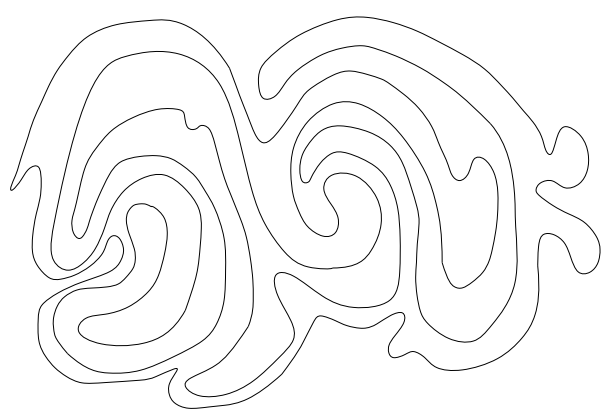

Figure $10 . A$ curve $\Gamma_{2}$ 


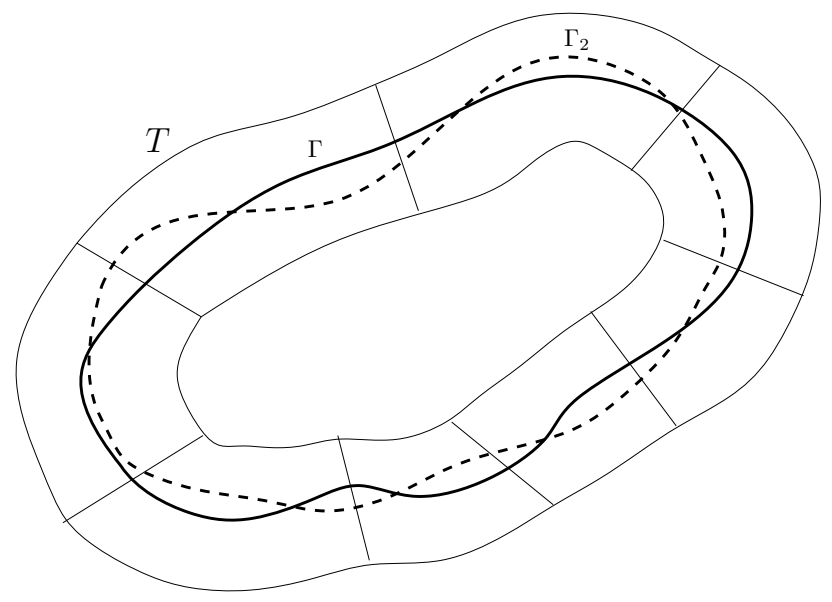

FiguRE 11

We now consider the smooth curve $\Gamma_{2}$. Its embedding in $\mathbb{R}^{2}$ may be rather complicated, with a lot of horizontal contact points, as it is suggested in Figure 10. In the rest of the proof we explain how to simplify this embedding.

If there are just two such horizontal contact points, a maximum and a minimum for the $y$ function, we can displace $\Gamma_{2}$ by an affine map such that the maximum is on the line $\{y=1\}$ and the minimum on the line $\{y=-1\}$. It is now very easy to construct a smooth diffeomorphism $G(x, y)=(g(x, y), y)$ sending $\Gamma_{0}$ onto $\Gamma_{2}$ (see Figure 12$)$

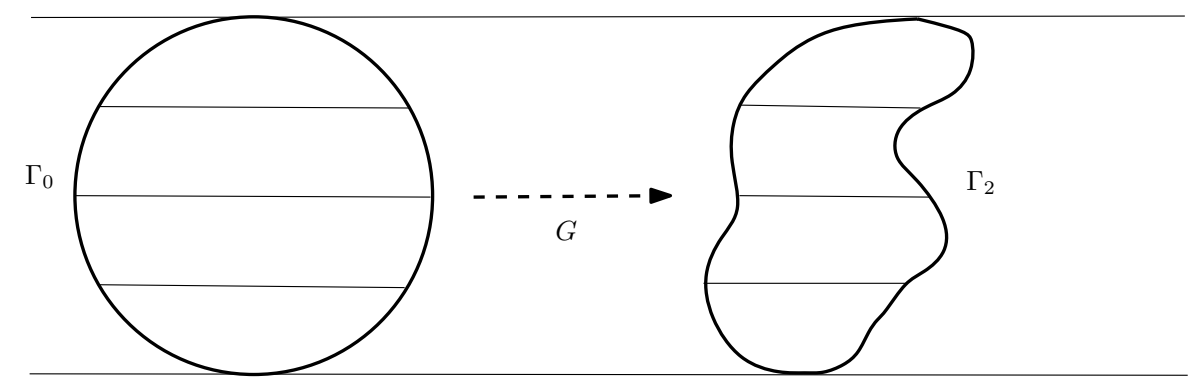

Figure 12. Curve $\Gamma_{2}$ with two contact points

If there are more than 3 contact points, it is possible to prove that there is at least a pair of two successive contact points on $\Gamma_{2}$, a minimum $p$ and a maximum $q$, in the position illustrates in Figure 13: there is a disk $B$ such that $\partial B$ is union of an $\operatorname{arc} \Gamma_{2}\left(p, p^{\prime}\right)$ on $\Gamma_{2}$ containing $q$ in its interior (and no other contact point) and the horizontal segment $\left[p p^{\prime}\right]$. Moreover, at $p$, the complement $\Gamma_{2} \backslash \Gamma_{2}\left(p, p^{\prime}\right)$ starts outside $B$. The disk $B$ may contain other parts of $\Gamma_{2}$ disjoint from $\Gamma_{2}\left(p, p^{\prime}\right)$. Let $\Gamma_{B}$ be their union. The existence of such a pair $(p, q)$ of contact points is the key point of the proof. It can be obtained by an easy recurrence argument on the number of contact points. We will not elaborate further on it. 
We now consider such a pair $(p, q)$ and explain how to eliminate it. We proceed in two substeps. First, we choose a disk $W$ disjoint from $\Gamma_{2}\left(p, p^{\prime}\right)$, and such that $\Gamma_{B}$ is inside $W \cap B$. Then, we push $\Gamma_{B}$ outside $B$ by a smooth diffeomorphism, with support in $W$ which, in a neighborhood of $\Gamma_{B}$, sends horizontal intervals into horizontal intervals located outside $B$ (see Figure 13).

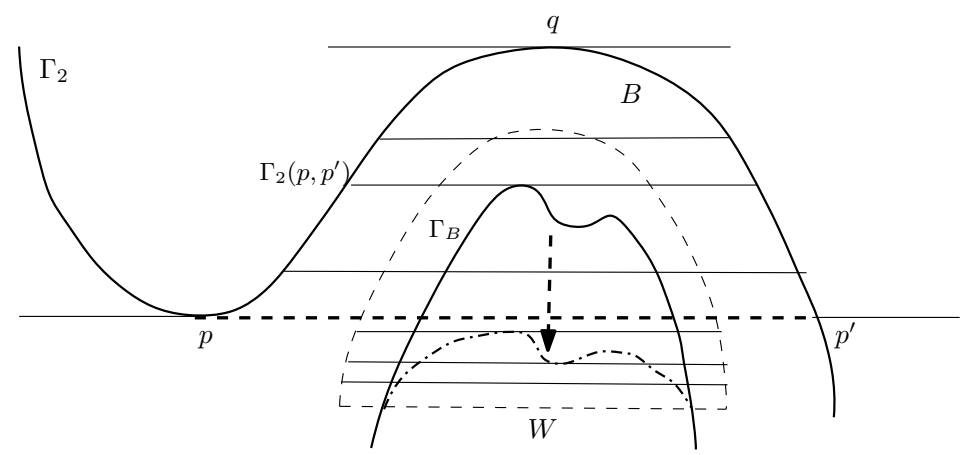

Figure 13. Cleanning out a disk B

We obtain a new curve $\Gamma_{3}$, in generic position and coinciding with $\Gamma_{2}$ in a neighborhood of $\Gamma_{2}\left(p, p^{\prime}\right)$, with the same number of horizontal contact points as $\Gamma_{2}$. But now the same disk $B$ is associated to $\Gamma_{3}$ and does not contain other parts of $\Gamma_{3}$ than $\Gamma_{2}\left(p, p^{\prime}\right)$. It is now easy to construct a diffeomorphism, with support in a compact neighborhood of $B$, which pushes the arc $\Gamma_{2}\left(p, p^{\prime}\right)$ downward outside $B$, in order to eliminate the pair $(p, q)$ without modifying the other contact points nor creating new ones (see Figure 14).

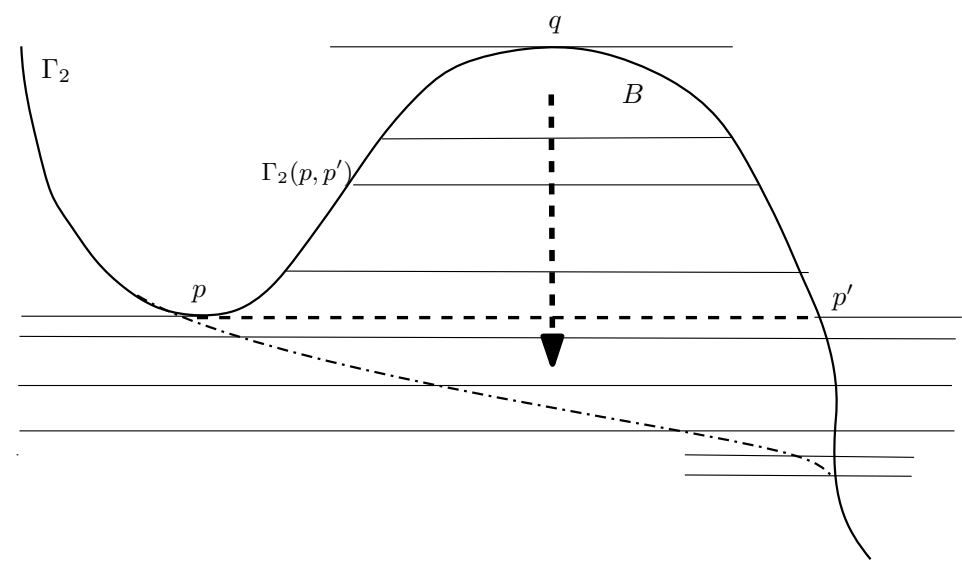

FiguRE 14. Elimination of the pair $(p, q)$

One can apply this argument by recurrence to finish with a curve which has just two contact points. Finally, we have obtain a sucession of diffeomorphisms of $\mathbb{R}^{2}$, with compact support and 
of class at least $\mathcal{C}^{1}$, whose composition (of them or their inverse) sends the trigomometric circle onto the given initial closed curve $\Gamma$.

\section{REFERENCES}

[1] I. Bendixson, Sur les courbes définies par des équations différentielles, Acta Math., 24 (1901), 1-88.

[2] C. M. Brauner, R. Roussarie, P. Shang, L. Zhang, Existence of a traveling wave solution in a free interface problem with fractional order kinetics, Preprint, Hal-02979187 (2020)

[3] S. S. Cairns, An elementary proof of the Jordan-Schoenflies Theorem, Proc. Amer. Math. Soc., vol 2, n6, (1951), 860-867

[4] Y.N. Dowker and F.G. Friedlander, On limit sets in dynamical systems, Proc. London Math. Soc (3) 4, (1954), 168-176.

[5] F. Dumortier, Singularities of vector fields on the plane, J. Diff. Equ. Vol 23, $n^{\circ} 1$, (1977), 53-106.

[6] F. Dumortier, J. Llibre and J.C. Artés, Qualitative Theory of Planar Differentiable Systems, Universitext, Springer-Verlag (2006), 15-31.

[7] S. Lefschetz, Differential equations: Geometric theory, $2^{\text {nd }}$ edition, Dover Publications Inc., New York, 1977.

[8] J. Milnor, Topology from the differentiable point of view, Princeton Landmarks in Math. Princeton University Press, New Jersey, (1997).

[9] J. Palis and W. de Melo, Geometric theory of dynamical systems, Springer, Berlin, Heidelberg, New-York (1992).

[10] D. Panazzolo and R. Roussarie, A Poincaré-Bendixson Theorem for analytic families of vector fields, Bol. Soc. Math. Bras., vol. 26, n 1 (1995) 85-116.

[11] H. Poincaré, Sur les courbes définies par des équations différentielles, Journ. de Math. Pures et Appl., s.3 t.VII (1881) $=$ [Oeuvres I, 3-44]; (Second part) Id., s. 3, t. VIII (1882) $=$ OOeuvres I, 44-84]; (Third part) Id., s. 4, t. I $(1885)=[$ Oeuvres I, 90-158]; (Fourth part) Id., s. 4, t. II (1886)=[Oeuvres I, 167-222], Oeuvres de Henri Poincaré, I-XI, Gauthier-Villars (new impression 1950-1965).

[12] R. Roussarie, A topological study of planar vector field singularies, a tribute to Ivar Bendixson, DCDS-A, Vol. 40, $n^{\circ} 9(2020), 5217-5245$.

Institut de Mathématiques de Bourgogne, UMR 5584 CNRS Université de Bourgogne-Franche Comté, B.P. 47870 F-2100-Dijon Cedex, France

Email address: Robert.Roussarie@u-bourgogne.fr 